\title{
Fuzzy logic for robust detection in wireless communications
}

\author{
A. Pérez-Neira, M.A.Lagunas, J.Bas ${ }^{1}$ \\ Dept. Teoria del Senyal i Comunicacions \\ ETSI.Telecom. Barcelona \\ Campus Nord UPC. Edificio D-5. c./ Jordi Girona, 1-3 \\ 08034 Barcelona- ESPAÑA
}

\begin{abstract}
This work addresses the problem of spatial reference estimation in mobile scenarios. Novel techniques based on fuzzy logic are introduced to enhance the performance of a tracking system. Specifically, the model-free function approximation capability of fuzzy logic is used to obtain high resolution angle estimates from the spatial spectral density. These estimates are used to improve the resolution of the tracker. To the authors knowledge it is the first time that fuzzy logic is introduced in array spectral estimation. This work also develops a fuzzy controller for acting as an interpolative supervisor of different trackers that apply in different operating conditions of the dynamic nonlinear system. The result is a localization and tracking system that attains a resolution comparable to that of high resolution techniques as the Minimum Variance or Capon estimator. One of the main features of the proposed technique is its low computational burden. In summary, the presented system supports the expectation of adaptive arrays for obtaining a communication front-end of affordable complexity, developing cost and good performance.
\end{abstract}

\section{INTRODUCTION}

In a recent work [1] the authors derive the Alternate Projection algorithm as a constrained phased array that is supposed to look at one source an block all the others. From this approach the authors solve the multiple source tracking problem as decoupled single source tracking problems; thus, reducing complexity. However, the concept of global tracker includes additional processing and data fusion, which enables to cope with eventual fadings of bounded time duration, as it may occur in crossing radial trajectories of two movils. That is why we introduce at each parallel branch i the additional processing outlined in figure 1

In the system proposed in [1], at each parallel branch the Spatial Power Density is measured after the constrained scanning. Thus resulting a so-called notch periodogram (system P). If no other users interfer in this periodogram its maximum corresponds to the desired user location. However, the resolution of the constrained scanning beam is limited by that of the array; thus, degrading the behaviour of the tracker for close users. The switch block detects this resolution threshold and smoothly commutes to the proposed fuzzy DOA estimator (system F), which produces the estimates for the two closely located users $(\theta \mathrm{i}, \theta \mathrm{j})$.

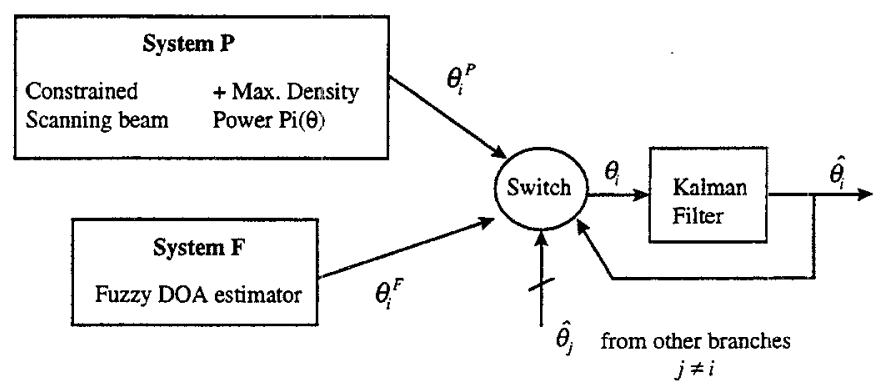

Figure 1. Proposed tracker at each parallel branch $i$

The computational burden of the presented tracker is low as fuzzy techniques just require a fixed number of products/ additions or a fixed number of comparisons/ additions; thus, accomodating to real time software or hardware implementation respectively.

First Section 2 formulates the problem. Next, section 3 presents the different fuzzy systems involved in the tracker of figure 1: the system $F$ for source location and the switch block. Finally, the simulations show the performance of both the fuzzy DOA estimator and the global tracker depicted in figure 1 . Both, synthetic and real data are considered.

\section{PROBLEM STATEMENT}

We address a digital wireless communication system employing adaptive arrays for the localization of NS moving users using an array of NQ identical radio receivers. The NS users operate simultaneously in the frequency and time slot. If we suppose that the antenna array consists of NQ sensors of known characteristics (calibrated array) and letting ai $(\theta \mathrm{k})$ be the gain and phase response of ith sensor in the direction $\theta \mathrm{k}$, then the signal output of the ith sensor at time $n$ will be

$$
z_{i}(n)=\sum_{k=1}^{N S} a_{i}\left(\theta_{k}\right) s_{k}(n)+n_{i}(n)
$$

where $\mathrm{ni}(\mathrm{n})$ is the noise generated at the ith sensor (usually thermal noise) and $\operatorname{sk}(\mathbf{n})$ is a scaled and phased-delayed

\footnotetext{
${ }^{1}$ This work has been supported by National Research Plan of Spain CICYT under grant number TIC-96-0500-C10-01
} 
version of the signal emitted by user $k$. The data zi(n) from all the NQ sensors are collected in the so-called snapshot vector $\mathbf{z}(\mathrm{n})$. In this model we have assumed that the distances between the mobiles and the base station are large; therefore, the local scatterers in the vicinity of the mobile have negligible radius in comparison with the distance between the base station and the receiver and the fading of the antenna elements can be assumed to be fully correlated. We note that although of limited applicability to real-world problems, this clutter-free model lies at the core of many viable algorithms.

Finally, in our work a small number of snapshots is necessary to carry out an angle estimate (less than 10 snapshots); thus, the relative array velocity does not change over the observation interval and can be assumed zero. The problem of interest is to estimate and track the NS sources' angular positions $\theta \mathrm{k}(\mathrm{n})$ from the data $z(n)$.

\section{LINGUISTIC-BASED KNOWLEDGE FOR ROBUST LOCALIZATION AND TRACKING.}

It is well-known the introduction of expert knowledge in time or spatial frequency tracking and control. Fuzzy systems [2] especially have gained prominance due to the surprising ease with which fuzzy controllers can be designed. However, although fuzzy systems are model-free approximators of any continuous function, relatively few applications have been made to estimation problems [3-4]. This paper focusses on this estimation capability of fuzzy systems and applies it to obtain high resolution DOA (Direction Of Arrival) estimates.

\subsection{Fuzzy logic function approximation for source localization}

By "observing" the periodogram $\mathrm{P}(\theta)$, the proposed fuzzy logic system produces DOA estimates even if the angle separation between two users is less than the resolution of the array. With suitable training pairs this simple fuzzy system can exactly map main lobe bandwidths into estimated source DOA's.

\subsubsection{Basic architecture of Fuzzy Logic System (FLS)}

The basis for a correct design and understanding of the system is the description of the input and output fuzzy variables (collected in vector $\mathbf{x}$ and $\mathbf{y}$ respectively). At each instant of time $\mathrm{n}$, the two inputs are the maximum of the Periodogram, $\theta \max$, and the main-beam normalized bandwidth, BW. As an answer to these two inputs, the FLS computes the distance, $d$, of each source to the measured maximum. The fuzzy system $F: X \rightarrow Y$ is a set of IF-THEN rules that map inputs into outputs, in our case, it approximates the function $\mathrm{f}(\mathrm{BW}, \theta \max )=\mathrm{d}$. Specifically, the additive fuzzy system $F$ that we are going to develop uniformly approximates the function $\mathrm{f}$ if $\mathrm{X}$ is compact and $\mathrm{f}$ is continuous [3].

At time $\mathrm{n}$ the fuzzy DOA estimates $\theta^{\mathrm{F}}$ of the two close sources $i$ and $j$ are

$$
\theta_{i}^{F}=\theta \max -d ; \quad \theta^{F}=\theta \max +d
$$

Figure 2 shows the architecture of the proposed high resolution DOA estimator. We note that the $10 \mathrm{~dB}$ main-lobe bandwidth values $\left(\delta_{\mathrm{p}}\right)$ for angles $\left[0^{\circ}, 10^{\circ}, 20^{\circ}\right]$ and NQ possible number of sensors should be stored in order to produce the normalized bandwidth BW from the measured one.

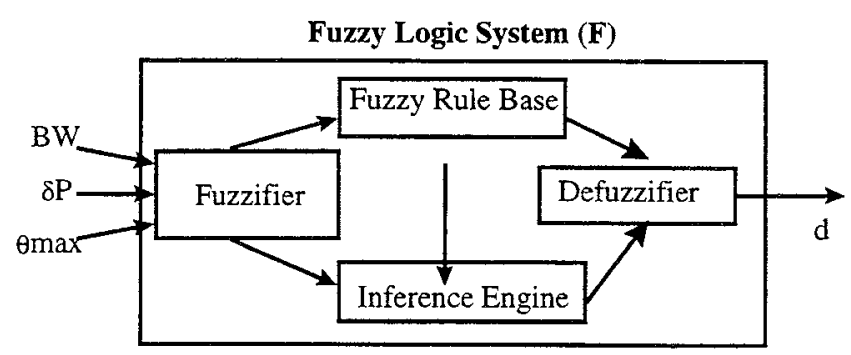

Figure 2. Proposed Fuzzy DOA estimator

The ranges of $\mathrm{BW}, \theta \max$ and $\mathrm{d}$ are as follows: $1 \leq B W<2 ;-25^{\circ} \leq \theta_{\max } \leq 25^{\circ} ; 0 \leq d<\delta_{P}$. The parameter $\delta_{\mathrm{p}}$ refers to the resolution of the periodogram. In the field of view $\left( \pm 25^{\circ}\right)$ the fuzzy system is able to resolve sources closer than $\delta_{\mathrm{p}}$.

Once the input and output variables have been picked, the basic configuration of additive FLS comprises the four principle components shown in figure $2:$ a) the fuzzifier, b) the fuzzy rule base, c) the fuzzy inference engine and d) the defuzzifier. This components are going to be described next.

A fuzzy set $A$ in a universe of discourse, $U$, is characterized by a membership function $\mu$ a, which takes values in the interval $[0,1]$; that is, $\mu_{A}: U \rightarrow[0,1]$. Thus, a fuzzy set $\mathrm{A}$ consists of a generic element $\mathrm{u}$ and its grade or membership function; that is, $A=\left\{\left(u, \mu_{A}(u)\right) \mid u \in U\right\}$. A fuzzy variable is characterized by a set of fuzzy sets, T(u) (i.e. of linguistic or fuzzy values) of $u$. In this work $A($.$) and x$ will be used for the input term set and the input variable, respectively. Also $B($.$) and y$ will be used for the output term set and the output variable, respectively. In our case the variable $\mathbf{x}$ is a vector of two components. For instance, if the component $x 1$ represents the location of the maximum $\theta$ max, its term set may be chosen as (very negative (A1(x1), negative (A2(x1), zero ( $A 3(x 1)$, positive $(A 4(x 1))$, very positive $(\mathrm{A} 5(\mathrm{x} 1))\}$.

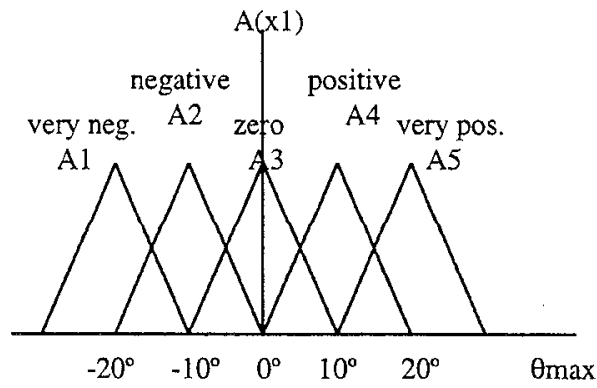

Figure 3. Membership functions for the fuzzy values of the maximum of the periodogram.

Each of these terms or fuzzy membership functions can have different shapes. The triangular shapes shown in figure 3 simplify computation. Later on we comment on the convenience of other shapes as Gaussian. Equally spaced membership functions as those in figure 3 are possible only if 
the mean values (centers) can be chosen by the designer. If these values are estimated by means of a training procedure, so that they adapt to the data that is associated with the rules (e.g. case of the input BW as described in sub-section 3.1.2), then unequally spaced functions are the norm.

The fuzzifier in figure 2 is a mapping from an input space to fuzzy sets in a certain input universe of discourse. The most widely used fuzzifier is the singleton fuzzifier. Because of its simplicity and lower computational requirements, it is the fuzzifier that we are going to use in this work. A singleton fuzzifier maps a specific value $x i(n)$ at time $n$ to the fuzzy set Al(xi) with degree $\mu_{A_{1}}(x i(n))$ and to the fuzzy set A2(xi) with degree $\mu_{A_{2}}(x i(n))$, and so on. We note, however, that in cases when the SNR (Signal to Noise Ratio) is low or there is high input uncertainty, non-singleton fuzzy sets as inputs can be used [5].

In fuzzy Logic theory, the behavior of a fuzzy system is characterized by a set of M IF-THEN rules $R_{m}$ that associate output fuzzy sets with input fuzzy sets. Our system is a twoinput/single-output system where the mth fuzzy rule is $R_{m}$ : $\left(A_{m}, B_{m}\right)$ or

$R_{m}: I F\left(x 1\right.$ is $A_{m}(x 1)$ and $x 2$ is $\left.A_{m}(x 2)\right)$

THEN (y is $\left.B_{m}(y)\right)$

Next section extracts these rules from numerical data. These associations are summarized in a bank of parallel fuzzy associative memory (FAM) [6] rules. We can easily construct, process and modify the FAM bank of FAM rules in software or in digital VLSI circuitry [7]

The inference engine in figure 2 is to match the preconditions of rules in the fuzzy system rule base with input state linguistic terms and to perform implication. The procedure of implication used to develop the fuzzy DOA estimator is the so-called the correlation-product inference [24]. Finally, before obtaining the DOA estimate, we need a defuzzification process to get a crisp value; the defuzzifier block in figure 2 serves this purpose. Among the commonly used defuzzification strategies, the center of area or fuzzy centroid-defuzzification methods yields a superior result. All centroidal fuzzy systems $F$ try to approximate the function $f($.$) with a conditional mean: f(x)=E[Y / X=x]$. Let $y j$ be the jth sample support value in the universe of discourse, $Y$, at which the membership function, $\mu(y j)$ represents its membership value. The defuzzification output (i.e. distance $d$ to the peridogram maximum $\theta \max$ ) is, therefore calculated by

$$
d(x, y)=\frac{\sum_{j} \mu(y j) y j}{\sum_{j} \mu(y j)}
$$

Notice that yj is called support value if $\mu(y j)>0$.

\subsubsection{Derivation of the Fuzzy Logic Rules.}

The selection of the fuzzy rules has a substantial effect on the performance of the FLS. The rules just translate the behaviour of the main lobe when two sources are closer than the array resolution $\left(\delta_{\mathrm{p}}\right)$. As this resolution depends on $\theta \max$ (i.e. there is more resolution in the broadside than in the endfire), a different set of rules is developed for each $\theta \max$.
To reduce computation the whole field of view or range of $\theta \max$ has been quantized in 5 values $\left[ \pm 20^{\circ}, \pm 10^{\circ}, 0^{\circ}\right]$ (figure 3 ). The fuzzy set values or terms associated with $x 1, \theta \max$, (i.e. $A j(x 1)$ ), have a triangular membership function centered at each of the quantized values.

To ellaborate the FAM's for each quantized $\theta$ max a set of $\mathrm{N}$ training pairs have been collected. In these training pairs the output, $\mathrm{d}$, has been quantized in $\mathrm{N}$ different values $\mathrm{di}=\mathrm{i}$ $(\mathrm{i}=0 \ldots \mathrm{N}-1)$. From these singletons the output membership functions $B(y)$ are designed. For each $\theta \max$, each distance di maps to the correspondig measured normalized bandwidth $B$ wi. These bandwidths are the support values to design the unequally spaced fuzzy set $\mathrm{A}(\mathrm{x} 2)$. Possible ambiguities are elliminated if Gaussian shaped membership functions are used in the set $A(x 2)$ instead of triangular shaped. Table 1 shows the $M=9$ rules for the case of a 9 antenna array. The ratio between the powers of both sources is considered to be less or equal to $7 \mathrm{dBs}$. This assumption is valid in communication systems with power control, where power groups can be allocated in time-frequency space such that their dissimilar power does not negatively affect the SDMA.

The FAM's table just associates the membership function of each training pair. Just by vector/matrix multiplications, each input fires parallely [6]. Note that rules just come from

\begin{tabular}{|c|c||c|}
\cline { 2 - 3 } \multicolumn{1}{c||}{} & \multicolumn{2}{c|}{$\begin{array}{c}\text { x2: Bandwith } \\
\text { (exponential) }\end{array}$} \\
\hline $\begin{array}{c}\text { y:Position } \\
\text { (x1:Center } \\
\left.{ }^{\circ}\right)\end{array}$ & 0 & 10 \\
\hline \hline 0 & 2.6120 & 2.8350 \\
\hline \hline 1 & 2.6675 & 2.8611 \\
\hline \hline 2 & 2.8014 & 2.9497 \\
\hline \hline 3 & 3.2159 & 3.2020 \\
\hline \hline 4 & 4.1200 & 3.6262 \\
\hline 5 & 4.9362 & 3.9246 \\
\hline \hline 6 & 5.2716 & 4.0317 \\
\hline \hline 7 & 5.5333 & 4.1195 \\
\hline \hline 8 & 5.8779 & 4.2285 \\
\hline \hline
\end{tabular}

Table 1. Rules from training pairs for an array of 9 antenna.

numerical data: the data establish the fuzzy sets that appear in the antecedents and consequents of the rules. The system surface is the relationship between the input variables and the output or, alternatively, between the antecedents and the consequents. Certain points on the control surface correspond to the exact rules, where the conjunction of the antecedents is unity for a specific rule and none of the other rules fire. Between these points, the fuzzy logic system interpolates the output. This interpolation can be made exact by using the analytic expression for the output (3). This expression transforms the fuzzy inference system into a functional equivalent adaptive network.

Other aspect is that the mapping in table 1 is only exact after computing the mean of the different $B W$ for each $d i=i^{\circ}$ and when the periodogram is estimated with a large number of snapshots. However, as our work focusses on DOA 
estimation for tracking, less than 100 (e.g. 10) snapshots are considered. For this reason and to improve the interpolation an LMS algorithm is used to learn the rules by learning the estimator parameters (i.e. width of the input membership functions and position of the output membership functions [3]). The rules are learned from varios periodogram realizations. For each realization $\mathrm{k}$ the rules and input/output fuzzy sets are adaptively designed in order to minimize the cost function

$$
\Psi=E\left\{\left[d_{a c t}-d(x(k), y(k))\right]\right\}
$$

where $d_{a c t}$ is the actual distance of the sources to the measured maximum of the peridogram. By initializing the system with the proposed FAM's in table 1, the convergence of the system considerably speeds up.

\subsubsection{Implementation of parallel FAM rules}

A decision table relating quantized measurements to crisp control actions can be generated off line using control rules in order to shorten the running time of the Fuzzy system. In other words, the calculations of fuzzification, correlationproduct inference, and fuzzy centroid defuzzification can be performed on a computer on the basis of the control rules. After the calculations, each error/error-change pair will have its corresponding control input values. The decision table is sotred in memory in the form of a lookup table. Basically, the decision table is based on the discretization of both a universe of discourse and its associated membership function. The number of quantization levels should be large enough to provide an adequate approximation and yet be small enough to save memory storage. The choice of quantization levels has an essential influence on how much resolution can be obtained.

\subsection{Fuzzy Logic for automated control}

The switch in figure 2 that commutes between the proposed fuzzy DOA estimator and the constrained scanning can be simply designed as a hard-decision threshold detector: "if the DOA distance between close sources is less than the array resolution, the DOA estimates are then produced by the fuzzy system". However, fuzzy systems are also highly suitable for automated control and fuse data from different controllers producing smooth transitions even in noisy scenarios. For this reason we implement the switch following the FLC theory. The rules for switching at branch of user $i$ are:

$$
\begin{aligned}
R^{m}: I F\left(\hat{\theta}_{i}-\hat{\theta}_{j}\right) \text { is } T^{m} \text { THEN } \theta_{i} \text { is } \theta_{i k}^{m} ; j, i & =1 . . \text { NS users }, j \neq i ; \\
m & =1 . . \text { M rules } ; k=1,2
\end{aligned}
$$

where $\left(\hat{\theta}_{i}-\hat{\theta}_{j}\right)$ measures the distance between the angle of user $i$ and that of user $j$ (produced at branch $i$ and at each of the other (NS-1) branches, respectively), $T^{\text {m }}$ are the membership functions defined as in figure 4. Finally, $\theta_{i k}^{m}$ is the DOA estimate produced by either the scanning beam $\left(k=1: \theta_{i, m}^{m}=\theta^{p}\right)$ or by the fuzzy estimator $\left(k=2: \theta_{i 2}^{m}=\theta^{F}\right)$.

The fuzzy inference process we have been referring to in Section 3.1 is known as Mamdani's fuzzy inference method, which it is the most commonly seen fuzzy methodology. However, when the output of each rule is a constant like in our case (i.e. crisp output membership functions $\theta_{k}$ ) a Sugeno inference method is more suitable

$$
\theta_{i}=f\left(\hat{\theta}_{i}-\hat{\theta_{j}}\right)=\frac{\sum_{m=1}^{M} T^{m t}\left(\hat{\theta_{i}}-\hat{\theta_{j}}\right) \theta_{i k}^{m}}{\sum_{m=1}^{M} T^{m}\left(\hat{\theta_{i}}-\hat{\theta_{j}}\right)}
$$

Because of the linear dependence of each rule on the system's input variables, the Sugeno method is ideal for acting as an interpolative supervisor of the two DOA estimators that apply in different operating conditions. We note that using product for inference and sum for combining the rules is mathematically equivalent to a Bayesian approach to decision making and control.

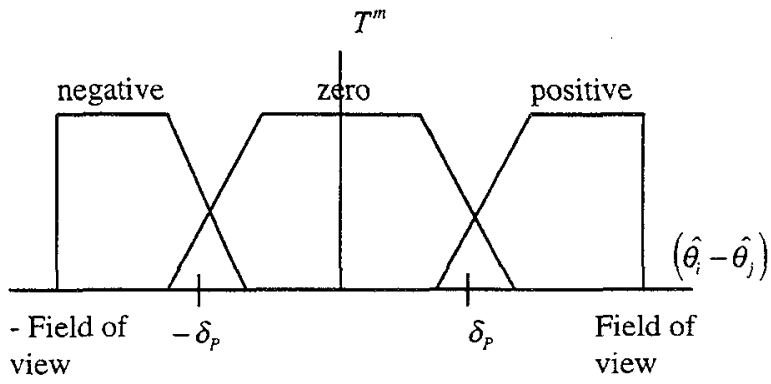

Figure 4. Membership functions for the fuzzy values of the maximum of the periodogram.

\section{SIMULATIONS}

The following simulations show the performance of both the fuzzy DOA estimator and the global tracker depicted in figure 1 . First, table 2 shows the DOA mean squared error ( 30 Monte Carlo runs) for two coherent sources of $S N R=5 \mathrm{~dB}$. The correlation matrix is computed from 100 snapshots and the fuzzy inference engine consists of 9 rules. The training positions $\left(i^{\circ} ; \mathrm{i}=0 \ldots 8\right)$ and the worst case of non-training positions (intermediate positions as: $0.5^{\circ}, 1.5^{\circ}$, etc.) have been measured. This table illustrates that the performance of the fuzzy DOA estimator is far closer to that of the Capon method than the performance of periodogram is. Next, in the same scenario than in table 2, figure 5 compares the performance the fuzzy DOA estimator and the Capon method when the training positions (i.e. the number of rules) are doubled but for $i^{\circ}=1^{\circ}\left(i^{\circ} ; i=0,2,2.5,3,3.5 \ldots 8\right)$. The reader can observe the improvement in the performance of the fuzzy system.

Next, figures 6 and 7 show the results after using some field trial data [8]. The scenario consists in 2 users of $30 \mathrm{~dB}$ each moving one each other with constant velocity. Figure 6 compares the performance of the periodogram estimates against the performance of the system proposed in [1] with parallel Kalman trackers. Figure 7 compares the actual source positions against the estimates obtained with the system proposed in figure 1 (parallel Kalman trackers with the fuzzy logic detection and estimation sub-systems). 


\section{REFERENCES}

[1] Pérez-Neira, A. and Lagunas, M.A. "High Performance DOA trackers derived from parallel low resolution detectors", 8thV IEEE Workshop on SSAP., pp. 558-561, Greece, June 1996.

[2] Zadeh, L.A., "Outlined of a new approach to analysis of complex systems and decision processes," IEEE Trans. O Systems, Man and Cybernetics, vol. 3, no.1, January 1973.

[3] Wang, L.X, Mendel, J.M., "Fuzzy adaptive filters, with application to nonlinear channel equalization," IEEE Trans. on Fuzzy Systems, vol. 1, no.3, pp. 161-170, August 1993.

[4] Kosko, B., "Fuzzy Engineering," Prentice-Hall, 1997.

[5] G.C.Mouzouris, J.M.Mendel, "Nonsingleton Fuzzy Logic Systems: Theory and Applications," IEEE Trans. on Fuzzy Systems, vol.5, nº 1, pp. 56-71, February 1997.

[6] B.Kosko, "Neural Networks and Fuzzy Systems," Prentice-Hall, 1992.

[7] IEEE Trans. on Fuzzy Systems, vol.4, no 4 , November 1996.

[8] TSUNAMI, "Report on the analysis of the field trial results," Doc. n: R2108/UPC/WP3.6/DR/P/035/b1, 1995.

\begin{tabular}{|c|c|c|c|}
\hline $\begin{array}{c}\text { Displacem } \\
\text { ent from } \\
\text { broadside } \\
\left({ }^{\circ}\right)\end{array}$ & $\begin{array}{l}\text { Fuzzy } \\
\text { Method }\end{array}$ & $\begin{array}{l}\text { Capon } \\
\text { Method }\end{array}$ & $\begin{array}{l}\text { Periodo- } \\
\text { gram }\end{array}$ \\
\hline 0 & 0 & 0 & 0 \\
\hline 0.5 & 0.2455 & 0.2339 & 0.2500 \\
\hline 1 & 0.0000 & 0.1011 & 1.0000 \\
\hline 1.5 & 0.2138 & 0.0178 & 2.2500 \\
\hline 2 & 0.0000 & 0.0068 & 4.0000 \\
\hline$\overline{2.5}$ & 0.2422 & 0.0034 & $\overline{6.2500}$ \\
\hline 3 & 0.0051 & 0.0019 & 9.0000 \\
\hline 3.5 & 0.2192 & 0.0046 & 12.2500 \\
\hline 4 & 0.0000 & 0.0064 & 16.0000 \\
\hline$\overline{4.5}$ & 0.2264 & 0.0104 & 19.8125 \\
\hline 5 & 0.0193 & 0.0129 & 0.6875 \\
\hline$\overline{5.5}$ & 0.1524 & 0.0172 & 0.7375 \\
\hline 6 & 0.0055 & 0.0106 & 1.5625 \\
\hline$\overline{6.5}$ & 0.1321 & 0.0088 & 1.5625 \\
\hline 7 & 0.0000 & 0.0060 & 1.3375 \\
\hline$\overline{7.5}$ & 0.0388 & 0.0384 & 0.6500 \\
\hline 8 & 0.0000 & 0.0015 & 0.3125 \\
\hline
\end{tabular}

Table 2. Power position error $\left({ }^{\circ} 2\right)$ ( 30 Monte-Carlo runs) The number of rules is 9 .

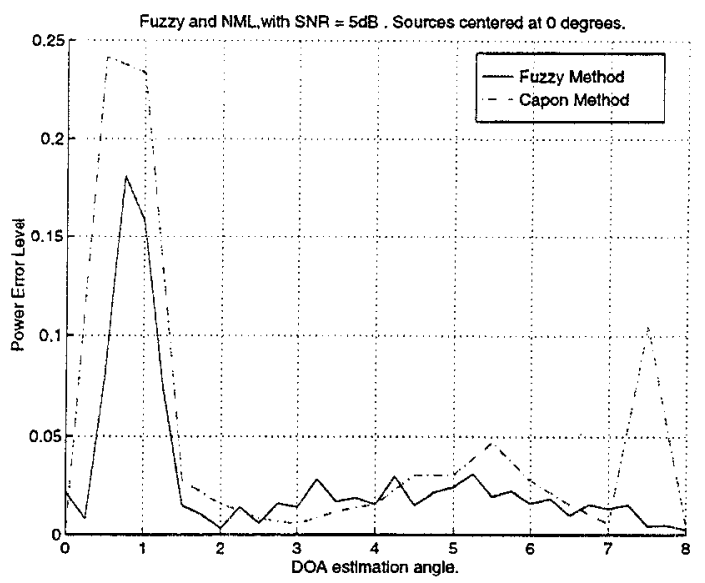

Figure 5. Power position error $\left({ }^{\circ} 2\right)$ (30 Monte-Carlo runs). The number of rules is 15.

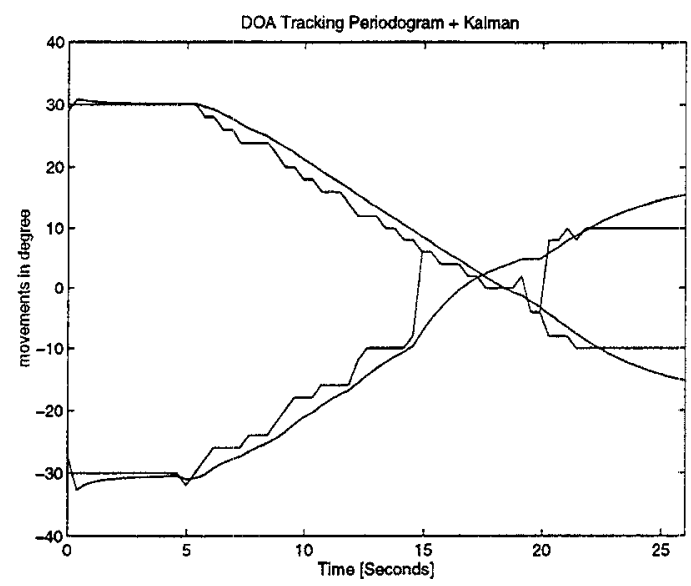

Figure 6.Field trial data.Periodogram against Kalman trackers

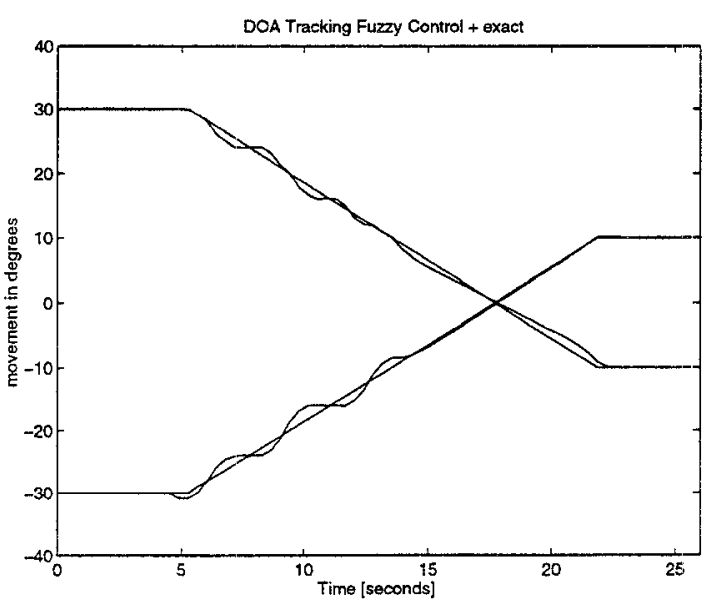

Figure 7.Field trial data.Actual DOA against fuzzy trackers 